\title{
Study and Research the Tensor of Dielectric Permittivity and Attenuation Transient in the Bended on Spiral Optical Fiber
}

\author{
AL-GAWAGZEH MOHAMMED YOUSEF, AL-HADIDI MOHAMMED RASOUL
}

\begin{abstract}
This paper investigates the assumption of spiralshaped index leads to an optimal result for the optical fiber modes. It also shows how to decrease the effect of optical fibers parameters such as attenuation and dispersion on the quality of transmitted signals, and to improve it into an acceptable form. The dielectric permeability tensor $\varepsilon_{i j}$ related to the curvature and torsion parameters $(\chi, U)$ in the coordinates system $(r, \varphi)$ was analyzed. The dependence of $\varepsilon_{i j}$ tensor on the bent on spiral optical fiber parameters was mathematically calculated. Also to study the exchange of power between the waves $H E_{11}^{o}$ and $H E_{11}^{e}$. The Transient attenuation dependence on spiral parameters of optical fiber with a length of (one kilometer) as a case study will be studied also.
\end{abstract}

Key-Words: - dielectric permittivity, Optical fiber, attenuation, wave power, spiral parameters.

\section{INTRODUCTION}

Currently, fiber optics transmission systems uses the properties of anisotropy which caused by mechanical stress (voltage), it's necessary to have full information about dielectric permittivity tensor characteristics, relationship between the ordinary wave $H E_{11}^{o}$ and extraordinary wave $H E_{11}^{e}$,photo elasticity effect on the basic parameters of optical fibers (attenuation and dispersion). to solve this problem, we require a comprehensive analysis for all properties of fibers under the influence of mechanical stress(voltage).

Optical anisotropy is the dependence of medium optical properties on the direction and polarization of wave propagation due to the medium dielectric or magnetic properties $[1,8,9,13,14]$.

The authors are with the Faculty of Engineering, Electrical power Engineering Department, Balqa Applied University, All- Salt JORDAN, gogazeh@bau.edu.jo,mohammad_hadidi@bau.edu.jo.
Many researches are related to this study, for example in $[2,3$, $4,5,6,7]$ the dependence of dielectric permittivity tensor $(\varepsilon)$ of spiral bent optical fiber on the step and radius of spiral was researched. Also, it was shown that during the transmit ion through the bent on spiral single- mode optical fiber (SSOF), the exchange of power between waves $\left(H E_{11}^{e}\right.$ and $H E_{11}^{o}$ ) was occurred, and this is the reason of appearance the attenuation and dispersion in the transmitted signal so there is no clear expressions to calculate the optimal value of spiral parameters during the transmission process for long distance with purpose of minimizing the effect of attenuation and dispersion on the quality of transmitted signal. the polarized dispersion was also studied.

The (SSOF) permittivity tensor dependence on the coefficient ( $A$ ) which shows the relationship between spiral parameters (curvature and spinning) and polarization angle of main waves ( $H E_{11}^{e}$ and $H E_{11}^{o}$ ), and the analysis of attenuation transient in the (SSOF) was not completely finished.

The purpose of this paper is to research the dielectric permittivity tensor, and attenuation transient due to power transfer between the waves $\left(H E_{11}^{e}\right.$ and $H E_{11}^{o}$ ) over the halfbeat length $\left(\zeta_{1}\right)$.and to research the attenuation transient of the bent on spiral optical fiber sections (for this study case, the wavelength range was selected between 1.565 up to 1.625 micron).

\section{METHOD AND MATERIAL}

The vectors $\vec{D}$ and $\vec{E}$, are related by the expression:

$$
D_{i}=\sum_{j=1}^{3} \varepsilon_{0} \varepsilon_{i j} E_{j},
$$


Where $\varepsilon_{i j}$ - Elements of dielectric permeability tensor.

$$
\varepsilon_{0} \text { - Electrical constant. }
$$

In work [5], the expression that describe the dependence of dielectric permeability tensor on the curvature and torsion of spiral optical fiber ( $\chi$ and $U$ ) was obtained according to the system of coordinates ( $x, y, z$ and $r, \varphi, z)$. and if we analyse this dependence only in the system of coordinates $(r, \varphi, z)$ Thus the dielectric permeability $\hat{\varepsilon}$ will be as next form $[5,6]$ :

$$
\hat{\varepsilon}=\left\|\begin{array}{lll}
\varepsilon_{r r} & \varepsilon_{r \varphi} & \varepsilon_{r z} \\
\varepsilon_{\varphi r} & \varepsilon_{\varphi \varphi} & \varepsilon_{\varphi z} \\
\varepsilon_{z r} & \varepsilon_{z \varphi} & \varepsilon_{z z}
\end{array}\right\|
$$

Where the elements $\varepsilon_{r r}, \varepsilon_{\varphi \varphi}$ and $\varepsilon_{z z}$ are defined by the ratios:

$$
\begin{aligned}
& \varepsilon_{r r}=\varepsilon_{\varphi \varphi}=\varepsilon(r) \\
& \varepsilon_{z z}=\varepsilon(r)-2 \chi r \cos \varphi+\chi^{2} r^{2} \cos ^{2} \varphi+v^{2} r^{2} .
\end{aligned}
$$

But the elements $\varepsilon_{r \varphi}=\varepsilon_{\varphi r}=\varepsilon_{r z}=\varepsilon_{z r}=0$, and then:

$$
\varepsilon_{\varphi z}=\varepsilon_{z \varphi}=-v r
$$

In equations $(3,4,5)$, the parameters $\chi$ and $v$ are determined as [5]:

$$
\chi=\frac{R}{R^{2}+(\rho / 2 \pi)^{2}}, v=\frac{\rho}{2 \pi} \frac{1}{R^{2}+(\rho / 2 \pi)^{2}}
$$

The physical sense of elements of dielectric Permeability tensor $\varepsilon_{i j}$ for the bent spiral optical fiber is related with elements of metric deformation tensor (due to the curvature and torsion of spiral)[7,8,9]. Thus the elements of $\varepsilon_{i j}$ that located on the main diagonal tensor (2) represent the distribution of dielectric permeability along $i$ axis which caused by mechanical deformations in this direction (compression or stretching). The positive values of deformation tensor components correspond to compression [5] which mean an increasing of $\varepsilon_{i j}$ in a direction of the given coordinate. and the stretching leads to reduction of the elements of $\varepsilon_{i j}$ along the given coordinate.

To research the dependence of $\left(\varepsilon_{i j}\right)$ on the bending parameters $(R, \rho)$ it is necessary to calculate $\left(\varepsilon_{i j}\right)$ elements when: $(\rho=50 ; 100 \mathrm{~mm}$, and $R=5 ; 7,5 ; 10 \mathrm{~mm})$ depending on the coordinates system $(r, \varphi)$, these calculation results are shown in table 1 .

Figure .1 shows, how the $\varepsilon_{z z}$ depend on $\varphi$ and other values of $\varepsilon_{i j}$ of optical fiber, when: $\lambda=1.55 \mu \mathrm{m}, R=5 \mathrm{~mm}$, $\rho=50 \mathrm{~mm}$. Thus the green curve (1) corresponds to coordinate $r=1 \mu \mathrm{m}$, and the blue curve (2) corresponds to coordinate $r=$ $2.5 \mu \mathrm{m}$. then $\varepsilon_{\varphi z}=9.009 \cdot 10^{-5}$ at $r=1 \mu \mathrm{m}$. and, $\varepsilon_{\varphi z}=$ $2.2523 \cdot 10^{-4}$ when $\mathrm{r}=2.5 \mu \mathrm{m}$

In General the $\varepsilon_{z z}$ dependence on the system of coordinates $(R, \rho, r, \varphi)$ can be written as :

$$
\varepsilon_{z Z}=\varepsilon(r) \pm \Delta \varepsilon
$$

$$
\Delta \varepsilon=-2 \chi^{2} \cos \varphi+\chi^{2} r^{2} \cos ^{2} \varphi+v^{2} r^{2}
$$

Where $\Delta \varepsilon$ depends on the parameters $(R, \rho, r, \varphi)$ only and does not depend on the wave length $\lambda$. 
Table.1: The Calculation dependence of dielectric tensor components on the coordinates $(R, \rho, r, \varphi)$

\begin{tabular}{|c|c|c|c|c|c|c|c|c|c|c|c|c|c|}
\hline \multirow{3}{*}{$\begin{array}{l}\dot{0} \\
\dot{0} 0 \\
\dot{0} \\
0 \\
0\end{array}$} & \multicolumn{4}{|l|}{5} & \multicolumn{4}{|l|}{7.5} & \multicolumn{4}{|l|}{10} & \multirow{3}{*}{$\begin{array}{l}R \mathrm{~mm} \\
\rho \mathrm{mm} \\
r \mu \mathrm{m}\end{array}$} \\
\hline & \multicolumn{2}{|l|}{50} & \multicolumn{2}{|l|}{100} & \multicolumn{2}{|l|}{50} & \multicolumn{2}{|l|}{100} & \multicolumn{2}{|l|}{50} & \multicolumn{2}{|l|}{100} & \\
\hline & 1 & 2.5 & 1 & 2.5 & 1 & 2.5 & 1 & 2.5 & 1 & 2.5 & 1 & 2.5 & \\
\hline 0 & -1.12 & -2.84 & -0.36 & -0.9 & -1.26 & -3.14 & -0.49 & -1.21 & -1.23 & -3.06 & -0.57 & -1.42 & \\
\hline 15 & -1.1 & -2.73 & -0.34 & -0.87 & -1.21 & -3.03 & -0.47 & -1.17 & -1.19 & -2.96 & -0.55 & -1.37 & \\
\hline 30 & -0.98 & -2.45 & -0.31 & -0.78 & -1.09 & -2.72 & -0.42 & -1.05 & -1.06 & -2.65 & -0.49 & -1.23 & \\
\hline 45 & -0.8 & -2.0 & -0.26 & -0.64 & -0.89 & -2.22 & -0.35 & -0.86 & -0.87 & -2.17 & -0.4 & -1.0 & \\
\hline 60 & -0.57 & -1.42 & -0.18 & -0.45 & -0.63 & -1.57 & -0.24 & -0.61 & -0.61 & -1.53 & -0.29 & -0.71 & \\
\hline 75 & -0.3 & -0.73 & -0.1 & -0.23 & -0.33 & -0.81 & -0.13 & -0.32 & -0.32 & -0.79 & -0.15 & -0.37 & \\
\hline 90 & 0 & 0 & 0 & 0 & 0 & 0 & 0 & 0 & 0 & 0 & 0 & 0 & \\
\hline 105 & +0.3 & +0.73 & +0.1 & +0.23 & +0.33 & +0.81 & +0.13 & +0.32 & +0.32 & +0.79 & +0.15 & +0.37 & \\
\hline 120 & +0.57 & +1.42 & +0.18 & +0.45 & +0.63 & +1.57 & +0.24 & +0.61 & +0.61 & +1.53 & +0.29 & +0.71 & \\
\hline 135 & +0.8 & +2.0 & +0.26 & +0.64 & +0.89 & +2.22 & +0.35 & +0.86 & +0.87 & +2.17 & +0.4 & +1.0 & \\
\hline 150 & +0.98 & +2.45 & +0.31 & +0.78 & +1.09 & +2.72 & +0.42 & +1.05 & +1.06 & +2.65 & +0.49 & +1.23 & \\
\hline 165 & +1.1 & +2.73 & +0.34 & +0.87 & +1.21 & +3.03 & +0.47 & +1.17 & +1.19 & +2.96 & +0.55 & +1.37 & \\
\hline 180 & +1.12 & +2.84 & +0.36 & +0.9 & +1.26 & +3.14 & +0.49 & +1.21 & +1.23 & +3.06 & +0.57 & +1.42 & \\
\hline
\end{tabular}

Table.1.show the symmetry of $\Delta \varepsilon$ referring to the angle $\varphi$ $=90^{\circ}$ but with an opposite sign. So in this table the values $\Delta \varepsilon$ are given only in the specified interval of $\varphi$ changes. 


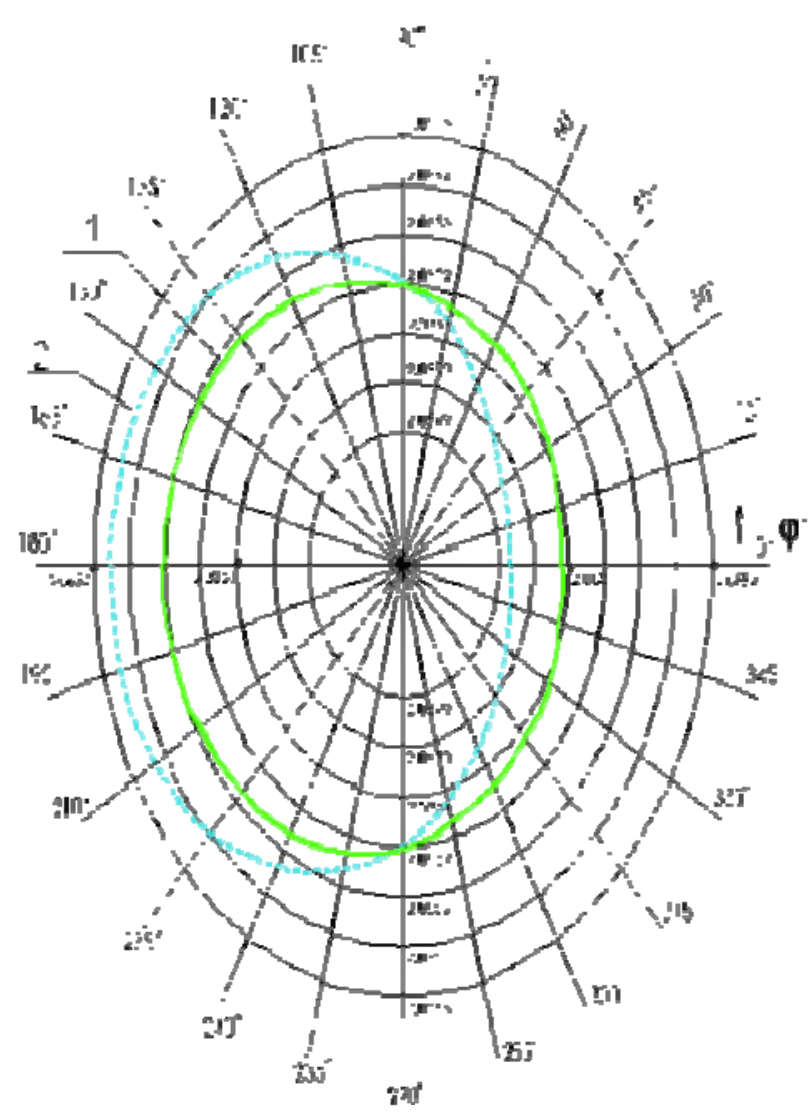

Fig .1: dependence of $\varepsilon_{z z}$ on $\varphi$

Analysis the elements of Dielectric tensor

The inclination angles for the waves $H E_{11}^{e}$ and $H E_{11}^{o}$ according to $(X)$ axes are rotating with angular velocity of winding spiral as follows:

$$
\varphi^{e}=-\operatorname{arctg} \frac{\rho}{4 \pi R} ; \varphi^{o}=\varphi^{e}+\frac{\pi}{2}
$$

Where: $R$-spiral radius, $\rho$-twisting step.

The power pumping direction is changing in opposite with equal intervals and called beat half-lengths $\left(\xi_{m}\right)$ which given by:-

$$
\xi_{m}=\frac{m \pi}{\left|\beta_{1}^{e}-\beta_{1}^{o}\right|}
$$

Where $\beta_{1}^{e}, \beta_{1}^{o}$-the spreading phase coefficients for the ordinary and extraordinary waves respectively. $m$-integer, which indicate the held quality of power moving to the optical fiber section. so if $m= \pm 1, \pm 3, \pm 5$ then the power will moving from $H E_{11}^{e}$ to $H E_{11}^{o}$ wave, and if $m= \pm 2, \pm 4, \pm 6$, the induced power in $H E_{11}^{o}$ will be pumped back to the wave $H E_{11}^{e}$.

Let analyse the dependence of tensor elements $\varepsilon_{i j}$ on the coefficient $A=\frac{\rho}{4 \pi R}$ in the $(r, \varphi, z)$ coordinates system. the diagonal tensor elements $\varepsilon_{r r}, \varepsilon_{\varphi \varphi}, \varepsilon_{z z}$ are defined as shown before from[4]as:

$$
\varepsilon_{r r}=\varepsilon_{\varphi \varphi}=\varepsilon(r)
$$

Where $\varepsilon(r)$-the dielectric permittivity of isotropic optical fiber.

$$
\varepsilon_{z z}=\varepsilon(r)-2 \chi r \cos \varphi+\chi^{2} r^{2} \cos ^{2} \varphi+v^{2} r^{2}
$$

Where the parameters $\chi$ and $v$ are the curvature and torsion of spiral axis $(\xi)$ and defined as:

$$
\begin{aligned}
& \chi=\frac{R}{R^{2}+\left(\frac{\rho}{2 \pi}\right)^{2}} \\
& v=\frac{\rho}{2 \pi} \cdot \frac{1}{R^{2}+\left(\frac{\rho}{2 \pi}\right)^{2}}
\end{aligned}
$$

In the work [6], the expressions for determining the curvature and torsion depending on the coefficient $(A)$ are as follow:

$$
v=\frac{2}{R} \cdot \frac{A}{1+4 A^{2}}
$$

$$
\chi=\frac{1}{R\left(1+4 A^{2}\right)}
$$

Substituting the expressions (15), (16) in the expression (12) and considering that $r=\frac{w}{\sqrt{2}}$,we get an expression for $\varepsilon_{z z}$ : $\varepsilon_{\mathrm{zz}}=\varepsilon(r)-\frac{\sqrt{2} w \cos \varphi}{R\left(1+4 A^{2}\right)}+\frac{w^{2} \cos ^{2} \varphi}{2 R^{2}\left(1+4 A^{2}\right)^{2}}+\frac{2 A^{2} w^{2}}{R^{2}\left(1+4 A^{2}\right)}$ 
Where $w$ - the modal field radius for the wave $H E_{11}$ in isotropic straight waveguide [6] and equal:

$$
w=\frac{a}{\sqrt{V-1}} ; V=\frac{2 \pi a}{\lambda} . N A
$$

Where $a$ - the optical fiber radius, $\lambda$-wavelength of transmitted signal, NA -numerical aperture of optical fiber.

The elements $\varepsilon_{z \varphi}$ and $\varepsilon_{\varphi z}$, can be represented as:

$$
\varepsilon_{z \varphi}=\varepsilon_{\varphi z}=-\frac{2 w}{\sqrt{2} R} \cdot \frac{A}{1+4 A^{2}}
$$

After calculations of $\varepsilon_{z z}(A)$, where $\varepsilon_{\varphi z}(A)=\varepsilon_{z \varphi}(A)$ with wavelengths( $1.565,1.595,1.625$ micron)of pure quartz material and coefficient ( $A$ )was chosen from( 0 to 1 )and spiral radius $R=2 \mathrm{~mm}$, these calculation results are plotted on figure. 2 and figure. 3 .

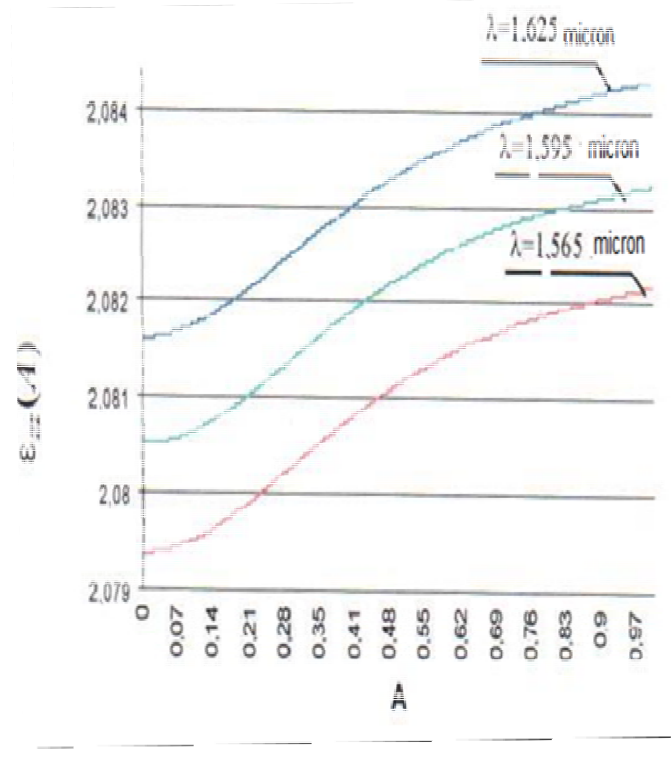

Fig. 2. Dependence of tensor element $\varepsilon_{z z}$ at $A$

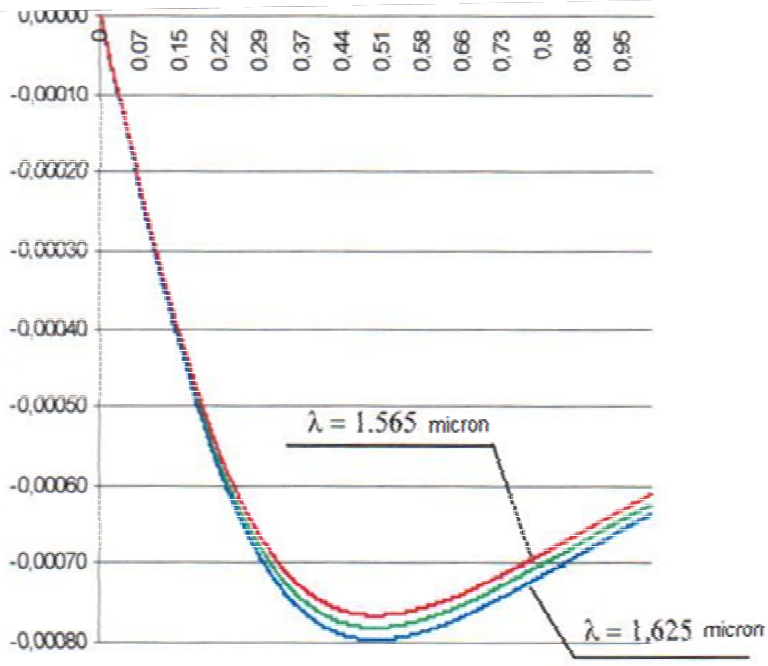

Fig. 3. Dependence of tensor elements $\varepsilon_{\varphi z}, \varepsilon_{z \varphi}$ at $A$

It is clear from the above figures that by increasing the value of ( $A$ ) value, the $\varepsilon_{z z}$ will tends to the value of dielectric permittivity of isotropic optical fiber $\varepsilon(r)$ and we can notice that for $\lambda=1.565$ micron $\varepsilon(r)$ $=2.084683$, and for $\lambda=1.595$ micron $\varepsilon(r)=2.083643$, and for $\lambda=1.625$ micron $\varepsilon(r)=2.082573$. and this leads to reduce the effect of second,third and fourth summands in expression (17).

According to expression (15), if $A>1$ then the curvature of optical fiber will be decreases, and the non-diagonal elements $\varepsilon_{\varphi z}$ and $\varepsilon_{z \varphi}$ will be decreasing also according to expression (5).

Consequently, by increasing ( $A$ ) coefficient, the diagonal elements of tensor are equal to $\varepsilon(r)$, and the non-diagonal element tends to Zero. and this mean that the characteristic of (SSOF) will be closer to isotropic.

\section{Attenuation Transitive in SSOF}

If we analysing the losses due exclusively anisotropy of optical fiber without attention to: scattering attenuation, resonance field absorption in the shell and core, radiation in the open space, the value of half-length beats can be calculated using the next expression $[6,10,11,12]$ :

$$
\xi_{1}=\frac{\sqrt{2} \lambda n_{1} R}{w} \cdot \frac{\left(1+4 A^{2}\right) \sqrt{1+A^{2}}}{\left|A^{2}+A-1\right|}
$$

The expression for calculating the transient attenuation when the power pumping from $H E_{11}^{e}$ wave into $H E_{11}^{o}$ wave on a plot of half-length beat $\alpha_{n 1}^{e o}\left(\xi_{1}\right)[6]$ : 
$\alpha_{n 1}^{e o}\left(\xi_{1}\right)=10 \lg \frac{\sqrt{2} \lambda n_{1}}{w} \cdot \frac{A \sqrt{1+A^{2}}}{\left|A^{2}+A-1\right|} \cdot\{\cdot\}^{e o}$

And conversely, from $H E_{11}^{o}$ wave into $H E_{11}^{e} \alpha_{n 1}^{o e}\left(\xi_{1}\right)[7]$ :

$\alpha_{n 1}^{o e}\left(\xi_{1}\right)=10 \lg \frac{\sqrt{2} \lambda n_{1}}{w} \cdot \frac{A \sqrt{1+A^{2}}}{A^{2}+A-1} \cdot\{.\}^{\text {oe }}$

where $\{.\}^{e o}$-the expression in the braces from equation (29) [6], $\{.\}^{o e}$ from (35) [7], these expressions are not listed here because of their unwieldiness.

The transient attenuations are the product of the function of spectral dependence of transient attenuation of major waves, bending geometry, and material polarization angles.

The expressions (20), (21), (22) are depend on dimensionless parameter $A$, and after passing the length of $2 \xi_{1}$, the power will be pumped from one wave to another and then pumped back to the initial wave.

So the losses for $1 \mathrm{~km}$ of the length will be:

$\alpha_{n 1}(1 \mathrm{~km})=\frac{1000\left(\alpha_{n 1}^{e o}\left(\xi_{1}\right)+\alpha_{n 1}^{o e}\left(\xi_{1}\right)\right)}{2 \xi_{1}}$

Based on expressions (20), (21), (22), the experimental calculations done using (MATLAB) and the results of calculation are shown on figures $(4,5)$.

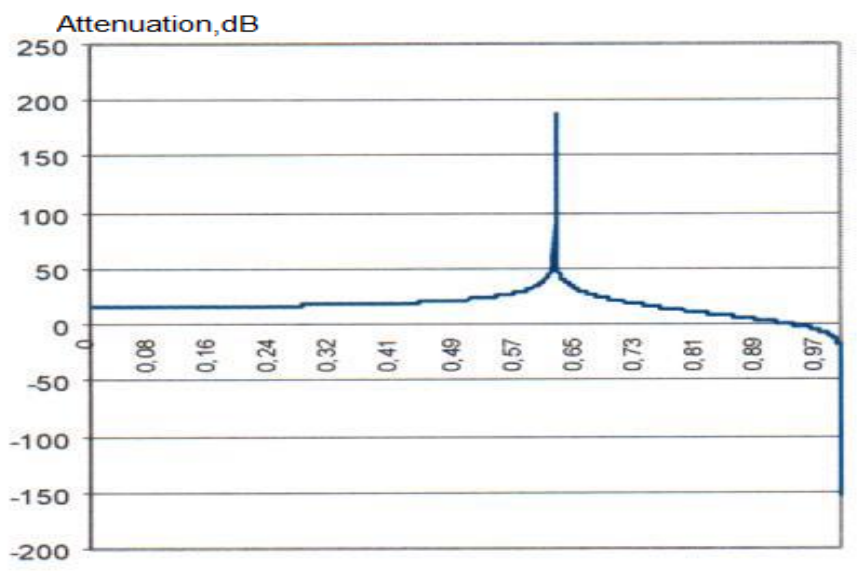

Fig. 4. Transient attenuation dependence with length of $2 \xi_{1}$ at $A$

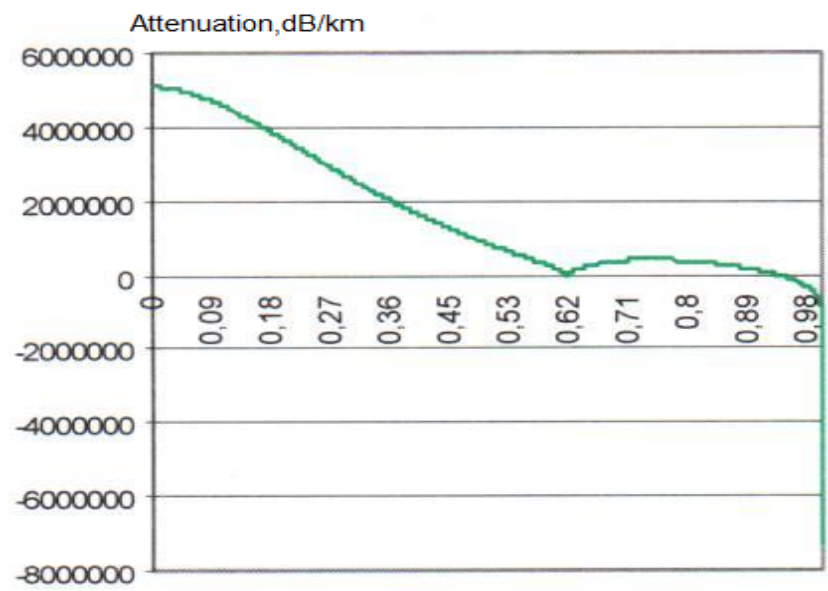

Fig.5. Transient attenuation dependence with length of (1 $\mathrm{km})$ at $A$

\section{RESULTS AND DISCUSSION}

$\Delta \varepsilon_{z z}$ Depends on coordinate's $r$ more than on parameters $\chi$ and $U$, which describe curvature and torsion. Also the big values of $\rho$ corresponds with the small values of $\Delta \varepsilon_{z z}$. With the changing of $R$ (from 5 to $10 \mathrm{~mm}$ ) and with the constancy of $r$ and $\rho$, the value of $\Delta \varepsilon_{z z}$ will increase no more than $30 \%$.

When $A \rightarrow 0.617999$, the value $\alpha_{n 1}\left(2 \xi_{1}\right) \rightarrow \infty$, and $\xi_{1}$ $\rightarrow \infty$, since the denominator in the expression (20) tends to zero due to the fact that the phase coefficients for ordinary $\left(\beta_{1}^{o}\right)$ and extraordinary $\left(\beta_{1}^{e}\right)$ waves are approximately equal to each other and their difference is the minimum, thus the material of (SSOF) is anisotropic. Therefore, the power over the length $(1 \mathrm{~km})$ has not time to pump from one wave to another. As the calculations shown the loss per $(1 \mathrm{~km})$ will be the minimum if $A \approx 0.618$ and $A>0.931$.and to provide a minimum transient attenuation, it is necessary that the value of $A$ must close to 1 ( $\rho \cong 4 \pi R)$.thus practically no power pumping flows from wave to another.

IV. CONCLUSION

Depending on equation (4) and calculated results in table. (1), we can change $\varepsilon_{i j}$ in specific limits, thus changing the transmission parameters to a better form.

And from the analysis of tensor elements of dielectric permittivity dependence on the coefficient $(A)$, we can also conclude that increasing the coefficient $(A)$, the effect of anisotropy will be reduced and it was shown for which values of $A$,the loss will be the minimum. Also, the transient attenuations were analyzed when the power pumped from one wave to another. 
Future work: these research results can be used to reduce the attenuation and dispersion effects on the transmitted signal by development the directional couplers, optical isolators, light modulators, dispersion compensators, which can implemented in $5 \mathrm{G}$ small cells networks, because with lower attenuation and immunity to electromagnetic interference the Optical fiber is the preferred medium.

\section{REFERENCES}

[1] Arnold, J.M, Anisotropic Effect in non-linear optical waveguides.J.of wave motion, 2001, V (34), No.3, pp.339-352.

[2] Makarov, T. V. Anisotropy of bending optical fiber. UNEERT 2, 2001, 103-108.

[3] Zhuck, N. P, Effective permittivity of a statistically inhomogeneous medium with strong permittivity fluctuations, Progress In Electromagnetic Research, PIER 44, pp.169-195, 2004.

[4] AL-Gawagzeh, M. Y. Research the Dielectric permittivity of optical fiber.J.of electronic and electrical engineering, 2009, 1(89), pp.45 -49.

[5] Al-Gawagzeh, M. Y..the effect of Anisotropy implementation on the components of optical transmission lines. IEEE Int. Conf. on information and Communication, 2004, pp.175-176.

[6] Makarov, T. V. nonreciprocal effect waves and signals in single mode bended on spiral optical fiber.UNEERT 1, 2004, pp. 23-29.

[7] Makarov,T. V. The transfer characteristic of bending optical fiber.UNEERT 2, 2004.pp.29-34.

[8] Snaider, A, Theory of optical waveguides (second Edition), Moscow, $2001,223 \mathrm{pp}$.

[9] Al-Gawagzeh, M. Y. AL-Hadidi, M. R. Rzooq,R. Polarized modes Dispersion in Anisotropic Optical Fiber Communication lines. J.of Wseas Transaction on Communication 8, 2009, pp. 11-20.

[10] Josselin G., High-frequency asymptotic for Maxwell's equations in anisotropic media Part Nonlinear propagation and frequency conversion, journal of mathematical physics volume 42, number 4 April 2001.

[11] V. G. Dmitriev, G. G. Gurzadyan, and D. N. Nikogosyan, Handbook of Nonlinear Optical Crystals $\sim$ Springer-Verlag, Berlin, 1991.

[12] Kornechok V. 1998. Optical Systems of Transfer, $3^{\text {rd }}$ ed., Radio and Communication Co., Kiev. 40.

[13] AL-Gawagzeh, M. Y. Studying the Polarization Dispersion In the Bended on Spiral Optical Waveguide $r$ Journal of Engineering and Applied Sciences 2018, 13(18), pp.4971-4975.

[14] Hussam Elbehiery. Optical fiber cables networks defects detection using thermal image enhancement techniques, WSEAS transactions on signal processing, 2018, Volume 14, pp.60-67. 